\title{
Application of the Modality of Multiple Disciplinary Team for a Perioperative Patient with Suspected Novel Coronavirus Pneumonia and Cervical Spine Fracture in Nursing
}

\author{
Yuanli Chen*, Yingying Zhang, Huijun Zhou, Wenzhen Li, Ruiting Hao, Liang Peng \\ The Third Affiliated Hospital of Sun Yat-sen University, Guangzhou, China \\ Email: *cyl.ff@163.com
}

How to cite this paper: Chen, Y.L., Zhang, Y.Y., Zhou, H.J., Li, W.Z., Hao, R.T. and Peng, L. (2020) Application of the Modality of Multiple Disciplinary Team for a Perioperative Patient with Suspected Novel Coronavirus Pneumonia and Cervical Spine Fracture in Nursing. International Journal of Clinical Medicine, 11, 769-777.

https://doi.org/10.4236/ijcm.2020.1112057

Received: November 23, 2020

Accepted: December 8, 2020

Published: December 11, 2020

Copyright $\odot 2020$ by author(s) and Scientific Research Publishing Inc. This work is licensed under the Creative Commons Attribution International License (CC BY 4.0).

http://creativecommons.org/licenses/by/4.0/

\section{(c) (i) Open Access}

\begin{abstract}
With the explosive spread of novel coronavirus pneumonia, a major public major public health emergency has been declared around the world. Our country has come to a crucial stage of "external defense input, internal defense rebound" and strict quarantine measures are taken in all ports of entry throughout the country. Operations on patients with cervical spine during the quarantine, which not only increases the risk of surgical treatment, but also increases the difficulty of perioperative nursing. The objective is to explore the result of application of the modality of multiple disciplinary team for a perioperative patient with suspected novel coronavirus pneumonia and cervical spine fracture in nursing. The patient's condition and nursing measures are studied and discussed from various specialist angle through the multidisciplinary team established by the isolation ward, orthopedics department, emergency department, anesthesiology department, operating room, rehabilitation department, psychology department and so on, and the overall and personalized surgical and nursing planning is formed through interdisciplinary advice. The nursing experience is summarized in this paper.
\end{abstract}

\section{Keywords}

Multiple Disciplinary Team, Suspected Novel Coronavirus Pneumonia, Cervical Spine Fracture, Perioperative Period, Nursing

\section{Background}

The pneumonia of unknown cause has aroused extensive attention from our society because it progressed quickly and people were generally susceptible. It was 
later identified by expert group of the National Health Commission as novel coronavirus pneumonia (hereinafter referred to as COVID-19) infection [1]. Droplet transmission and close contact transmission are the main routes of transmission and people are generally susceptible. The main symptoms of novel coronavirus pneumonia are fever, dry cough and fatigue and a few patients have nasal congestion, runny nose, sore throat diarrhea and other symptoms [2]. Although the prevention and control situation of COVID-19 has gradually improved in our country, the international situation is still breaking out and the importation situation is still grim. Special attention should be paid to preventing and controlling overseas input. One patients with COVID-19 and cervical spine fracture from abroad was admitted to our department. The patient has passed medical observation for further rehabilitation through close isolation and monitoring, timely surgical plan and proper nursing measures.

Multiple disciplinary team (hereinafter referred to as MDT) originated in the 1990s and was first proposed by the medical expert group in the United States. It refers to the clinical diagnosis and treatment mode of personalized diagnosis and treatment scheme for a certain system or organ disease through regular, fixed time and address specific meetings, which is generally composed of experts from two or more related disciplines. The purpose of this study is to provide patients with the most effective, minimal adverse reactions and the best quality of life on the basis of multidisciplinary demonstration. MDT reasonably and effectively utilizes and combines medical resources, which not only maximizes the advantages of medical personnel in various disciplines and greatly improves the therapeutic effect, but also improves the quality of medical service, patient satisfaction, the treatment efficiency and success rate of patients, and ensures the safety of patients [3].

\section{Clinical Data}

The 31-year-old male patient with incomplete paraplegia caused by a traffic accident started from Tanzania (epidemic area) on July 25, 2020 by the first-aid charter plane organized by International SOS organization, and arrived in Guangzhou through several countries. After arriving in Guangzhou, the negative pressure ambulance and medical staff of our hospital took him back to the isolation ward according to the requirements of secondary barriers.

The patient had repeated low fever on July 3 , and the fever subsided on July 10. The nucleic acid results of COVID-19 detected in the local hospital of Tanzania are shown in the Table 1 below.

The patient developed numbness of limbs and limited movement after a traffic

Table 1. The nucleic acid results of COVID-19 detected in the local hospital of Tanzania.

\begin{tabular}{ccccc}
\hline Date & 3 July & 10 July & 16 July & 20 July \\
\hline Nucleic acid test results & positive & negative & negative & negative \\
\hline
\end{tabular}


accident one month ago, whose both lower extremities are much more severe and his left side was better than his right side. His hands and wrists were weak and could barely move. His lower limbs could not move, stand or walk. He was sent to the local hospital in Tanzania for treatment after the injury, but things didn't get better after a period of time treatment. Then he was admitted to our department for further diagnosis and treatment. He had no unconsciousness disorder, no coma, good spirit, body weight loss about $5 \mathrm{~kg}$, uncontrolled defecation, indwelling catheter and good sleep after the injury. The nucleic acid and antibody results of COVID-19 were detected once he had been admitted to our hospital and both results were negative and all examinations were perfected. The results of CT and MR showed "C6 vertebral fracture, C5-7 vertebral arch fracture, C6 level spinal cord edema”. Lower spinous process and vertebral column existed obvious tenderness by physical examination. The shallow sensation (warm pain sensation) below $\mathrm{C} 4$ level was weakened, while the deep sensation existed. The muscle atrophy of both upper limbs was evident, but the muscle tone was normal. The muscle strength of elbow flexion and extension of both upper limbs was grade 5, that of wrist extension and wrist extension was grade 3 , that of finger flexion and abduction was grade 0 . The muscle atrophy of both lower limbs was slight, and the muscle tone was subdued. The muscle strength of left lower limb dorsum extensor was grade 4 and that of other two lower limbs was grade 0 . The primary diagnosis was "spinal cord injury (C6 level), spinal fracture (C4-6), acute incomplete quadriplegia”. On July 26, 2020, the patient underwent “anterior C6 vertebral subtotal resection+ anterior spinal canal decompression+ titanium mesh bone graft reconstruction+ plate and screw internal fixation" under general anesthesia. After the operation, the patients were treated with neck fixation, inhibition of postoperative inflammation and edema, inhibition of gastric acid, nutritional nerve, rehabilitation physiotherapy and functional training. The patient was in stable condition and was transferred to rehabilitation department for further rehabilitation treatment on August 8, 2020.

\section{Multidisciplinary Cooperation Mode}

\subsection{Build a Team}

It was a suspected COVID-19 patient who returned to China from the epidemic area and had cervical spine fracture that required surgical treatment and postoperative rehabilitation, which involved many disciplines, such as emergency transportation, emergency, orthopedics, anesthesia, surgery, rehabilitation exercise. Our hospital is a large 3 a general hospital which is also a provincial designated hospital to treat patients with COVID-19. We immediately set up a multi-disciplinary cooperation team, including isolation ward, orthopedics, emergency department, anesthesiology department, operating room, rehabilitation department, psychology department and other related medical personnel after receiving the task. The responsibilities of team members are shown in the Table 2 [4]. 
Table 2. The responsibilities of team members.

\begin{tabular}{|c|c|}
\hline member & duty \\
\hline $\begin{array}{l}\text { Emergency } \\
\text { Department }\end{array}$ & Transfer the patient and make handover with the staff of isolation ward \\
\hline Isolation Ward & $\begin{array}{l}\text { Admission education, preoperative preparation, observation and nursing of } \\
\text { postoperative complications, postoperative guidance of early rehabilitation } \\
\text { exercise, timely inform orthopedic doctors on duty when abnormal conditions } \\
\text { are found }\end{array}$ \\
\hline $\begin{array}{l}\text { Anesthesiology } \\
\text { Department }\end{array}$ & $\begin{array}{l}\text { Complete preoperative anesthesia visit, evaluate operation risk, formulate } \\
\text { specific anesthesia scheme, communicate with the patient's family }\end{array}$ \\
\hline $\begin{array}{l}\text { Operation } \\
\text { Room }\end{array}$ & $\begin{array}{l}\text { Prepare the negative pressure operating room, check and adjust the } \\
\text { intraoperative materials one day in advance, evaluate the operation position } \\
\text { together with the anesthesiologists and orthopedics personnel, and place the } \\
\text { patient's position carefully }\end{array}$ \\
\hline $\begin{array}{l}\text { Orthopaedics } \\
\text { Department }\end{array}$ & $\begin{array}{l}\text { Preoperative discussion to determine the surgical plan and implementation of } \\
\text { surgery, daily medical rounds, guidance of isolation ward staff to do a good job } \\
\text { in early rehabilitation training }\end{array}$ \\
\hline $\begin{array}{l}\text { Rehabilitation } \\
\text { Department }\end{array}$ & $\begin{array}{l}\text { According to the postoperative situation, the individualized rehabilitation } \\
\text { program was formulated, and early physical exercise and rehabilitation therapy } \\
\text { were performed }\end{array}$ \\
\hline $\begin{array}{l}\text { Psychology } \\
\text { Department }\end{array}$ & $\begin{array}{l}\text { According to the demands of patients, targeted psychological counseling, } \\
\text { convey greetings from relatives and friends, and enhance the rehabilitation } \\
\text { confidence of patients }\end{array}$ \\
\hline
\end{tabular}

\subsection{Implementation Plan}

\subsubsection{Standardized Personnel Training}

After receiving the task of treating the wounded, our medical workers in isolation ward immediately entered the preparation state and improved the training contents, including learning of inpatient environment, use of protective equipment, accountable and overall nursing care plan, related knowledge of the COVID19 , treatment plan, nursing measures and so on [4]. We participate in a week of theoretical and operational training in the orthopedics department, and all the staff were proficient in the key contents such as axis turning method, neck brace fixation, breathing and limb function exercise, and postoperative evaluation and observation. At the same time, medical workers confirmed that the articles in the isolation ward were in emergency and complete state, and determined the inspection route, transportation and operation route, and the main contents of succession.

\subsubsection{Preoperative Case Discussion}

On the day of admission, the orthopedic doctors worked out the operation method and date based on the results of various examinations. The anesthesiologist completed the preoperative visit and evaluated that the general and endotracheal intubation conditions were good. In the morning of the operation day, after the operation team checked the patients in bed, the operation, anesthesia and nursing teams conducted preoperative discussion. The surgeons and anesthesiolo- 
gists talked with the family members of the patient after the unified plan. Family members sign the relevant informed consent. The medical staff in the isolation ward completed preoperative fasting and water deprivation, skin preparation, blood matching, oral care, skin care, urethral care, prepared surgical handover record sheet and necessary instruments, and sent the patient to the operating room.

\subsubsection{Identification and Intervention of Psychological Problems}

The uncertainty of illness and social isolation are the main reasons for the patients to produce a series of emotional reactions after the patient enter the isolation ward, and the strong emotional reaction causes the body to produce stress response, which is easy to cause anxiety, impatience, pessimism and other negative emotions, resulting in the disorder of autonomic nerve function, the reduction of human immunity, and harmful effect on the outcome and prognosis of the disease [5]. Psychological doctors consult in time, conduct psychological counseling, and explain that rehabilitation training is a gradual process. Under the guidance of medical staff, active exercise can achieve good results. Nursing staff encourage the patient to express his thoughts, listen patiently to the needs, introduce the successful rehabilitation examples of similar diseases in the past, enhance the confidence in rehabilitation, make him actively cooperate with treatment, and improve his psychological disease resistance ability. Nurses should try to communicate with the patient and his families, friends, leaders and other relevant personnel to meet the needs of him as much as possible, so that he can feel the care of his families and friends [6].

\subsection{Giving Full Play to the Advantages of Multidisciplinary Team Work on Key Nursing Problems}

\subsubsection{Disinfection and Isolation Measures}

The patient returned from Tanzania and had low fever symptoms before, who was isolated as a suspected case according to the epidemiological investigation. After admission, the patient entered the isolation ward from the special channel and treatment was carried out in a single isolation room. Cleaning workers trained by professional personnel carried out cleaning and disinfection work in the isolation ward. Nurses guided the patient to pour $20,000 \mathrm{mg} / \mathrm{L}$ chlorine-containing disinfectant into the bedpan for 2 hours after each defecation and urination, and then pour them into the toilet. Relevant guidelines were formulated to inform the patient. The patient must wear a mask during hospitalization, and nurses also guided the wearing method of the mask and assisted the patient to wear it correctly. The air disinfector runs continuously for 24 hours [7].

\subsubsection{Disease-Related Care Measures}

After admission, closely monitor and record the vital signs, limb muscle strength, feeling, movement and defecaton and urination, pay attention to the occurrence of hypotension and hyponatremia, and monitor the urine volume and urine specific gravity. Focus on whether the patient has the following clinical symptoms 
and complaints: 1) Whether the patient has fever, fatigue, etc. 2) Patients with pneumonia: cough, expectoration, chest tightness, shortness of breath. 3) Patients with nasal congestion, runny nose, sore throat and diarrhea and other symptoms. 4) Whether the patient has dyspnea or hypoxemia, whether there is cyanosis in the nail bed and lips. 5) Whether the vital signs of patients are stable. Report to the doctor in time in case of any abnormality [7].

\subsubsection{Functional Exercise}

The principle of functional exercise is to start as early as possible, step by step, combine the active and passive, and persist for a long time. Without affecting the fixation, the muscles, tendons, ligaments and joint capsules of the affected limb should be recovered as soon as possible [8]. Specific rehabilitation exercise prescriptions were formulated by the orthopedic nursing staff after the ward round, which were assisted by nursing staff in the isolation ward. During the exercise process, the rehabilitation specialist carried out physical therapy such as lowfrequency pulse therapy, and the muscle strength was better than before.

\subsubsection{Intermittent Catheterization and Urination Training [9]}

Early intermittent catheterization is an important way of bladder training, and is the "gold standard" to assist bladder emptying. Intermittent filling and emptying of bladder contribute to the recovery of bladder reflex. Intermittent catheterization should be started as soon as possible after the patient's vital signs are stable. The specific method is to open the catheter every 4 hours and adjust the amount of drinking water according to the urine volume. After training, the patient has regained the consciousness of urination, and the inflow and outflow has reached a relatively balanced level, so as to pull out the catheter as soon as possible.

\subsubsection{Constipation}

After spinal cord injury, the nervous function of intestinal tract is damaged, and the colonic peristalsis is decelerated, so that the water absorption is more. In addition, the patient's activity and water intake is reduced, and the stool is harder and more difficult to expel. After the rounds of the nursing staff in the rehabilitation department, massaging the area surrounding anus to pull the anal sphincter is performed [10]. The specific method is to make the patient to take the left lying position and mat treatment towel under his hip, apply paraffin oil to the nurse's index and middle finger, and then stick slowly the index finger into the anus after massage. Next Expand the anus in the directions of 12, 3, 6 and 9 o'clock (suppose the patient's spine is in the direction of the clock at 12 o'clock), 10 times in each direction (take out when there is stool in the process of anal dilatation), and then massage clockwise. At the same time, the patient is instructed to eat more food rich in dietary fiber, fresh fruits and vegetables. The result is that the patient can exclude stool.

\subsubsection{Prevention of Pressure Sores}

Intermittent decompression can well prevent pressure sores [11]. The applica- 
tion of local decompression and various decompression equipment is the key. An air cushion bed was used immediately upon admission. Keeping the bed clean and dry, and turning over regularly during the bed rest every 2 hours is also necessary. We also put the patient in the correct position, change the weight support frequently to reduce the pressure, and use soft pillow, sponge pad and other protective equipment [12]. The axis turning method is adopted when turning the body. One needs to fix the head to make it turn with the shoulder at the same time. Before wearing the neck support, the Anpu patch was applied to the inner side of the neck bracket to reduce the friction force when contacting with the skin. The sacral, heel, iliac crest and other pressure parts were externally coated with Saifurun, and the skin of the patient was well protected.

\subsubsection{Cancel Quarantine}

The nucleic acid results of COVID-19 for three consecutive times within 14 days after isolation in our hospital were negative (shown in the Table 3 below), and the patient did not have respiratory tract and other related symptoms, which met the conditions for removing isolation. The medical staff informed the patient of the relevant attention points and knowledge of epidemic prevention and control. The patient was recommended to be transferred to the rehabilitation department for further rehabilitation treatment. The patient in rehabilitation department received treatment such as nerve nutrition, improving circulation, symptomatic relief and supportive treatment, passive joints movement training, hand function training, standing bed training, bladder training, electric acupuncture treatment. His finger flexion and extension abductor strength is restored to level 2, hip flexion strength is restored to level 3 , and knee extensor strength is restored to level 4. He could also turn over to both sides independently, and could sit up with a lot of help. The balance of the sitting position could reach level 2. Things are getting better and better.

\section{Discussion}

At present, the number of newly confirmed cases in several provinces of China has been growing at zero for many days. Great progress has been made in the prevention and control of the COVID-19 in China [13]. Although the risk of an outbreak is low at home, the global COVID-19 situation is still severe. The total number of confirmed cases is over 36 million, and more than 1 million deaths are reported. China is still facing high risk of imported cases. Therefore, prevention and control should not be relaxed under normal circumstances. "External defense input, internal defense rebound" has become the focus of epidemic prevention at present.

Table 3. The nucleic acid results of COVID-19 for three consecutive times within 14 days after isolation in our hospital.

\begin{tabular}{ccll}
\hline Date & 25 July & 1 August & 7 August \\
\hline Nucleic acid test results & negative & negative & negative \\
\hline
\end{tabular}


We apply the multidisciplinary collaboration model throughout the whole nursing process according to active and effective disinfection and isolation measures and perioperative nursing care, especially in observing the changes in the patient's condition, Identification and intervention of psychological problems, rehabilitating exercise, prevention of complications. We have achieved good results, which provide effective experience in admitting patients with COVID-19 and combined with many other diseases or complication. The application of multidisciplinary collaboration model not only improves the treatment effect, reduces the incidence of postoperative complications, improves the satisfaction of patients in hospital, but also promotes the development of nursing teams in various specialties.

\section{Acknowledgements}

This study was supported by grants from Tackling of key scientific and emergency special program of Sun Yat-sen University (SYSU-TKSESP) and emergency special program for 2019-nCoV of Guangdong province science and technology project (GDSTP-ESP) (2020B111105001).

\section{Conflicts of Interest}

The authors declare no conflicts of interest regarding the publication of this paper.

\section{References}

[1] World Health Organization. Surveillance Case Definitions for Human Infection with Novel Coronavirus (nCoV). https://apps.who.int/iris/handle/10665/330376

[2] National Health Commission, National Administration of Traditional Chinese Medicine (2020) Guidelines for the Diagnosis and Treatment of Coronavirus Disease 2019 (Trial Version Eighth). Chinese Journal of Viral Diseases, 10, 321-328.

[3] Mathoulin-Pelissier, S., Chevreau, C., Bellera, C., et al. (2014) Adherence to Consensus Based Diagnosis and Treatment Guidelines in Adult Soft-Tissue Sarcoma Patients: A French Prospective Population-Based Study. Annals of Oncology: Official Journal of the European Society for Medical Oncology, 25, 225-231. https://doi.org/10.1093/annonc/mdt407

[4] Cheng, L.L., Wang, J.J. and Xie, L. (2020) Application of Multidisciplinary Collaborative Nursing Mode in Severe Patients with Novel Coronavirus Pneumonia. Nursing of Integrated Traditional Chinese and Western Medicine, 6, 127-129.

[5] Yuan, B. and Liu, J. (2003) Psychological Problems and Therapy of SARS Patients. Chinese Journal of Nursing, 38, 418-419.

[6] Zhou, Y.C., Xia, C.L., Bai, X.N.,et al. (2017) Assessment of Stress Psychological Status and Coping Strategies of Patients with Traumatic Fracture Disability. Nursing Practice and Research, 14, 132-134.

[7] Dai, S.M., Li, Y.H., Li, S.H., et al. (2020) Nursing Care for Suspected COVID-19 Patients Admitted to the Infection Department of General Hospital. World Latest Medicine Information, 20, 227-228.

[8] Yao, C.Y. and Xu, S.F. (2011) Nursing Care of 36 Cases of Cervical Spine Fracture Combined with High Paraplegia. Chinese General Nursing, 9, 2861-2862. 
[9] Liao, L.M., Wu, J., Ju, Y.H., et al. (2013) Guidelines for Urinary System Management and Clinical Rehabilitation in Patients with Spinal Cord Injury. Chinese Journal of Rehabilitation Theory and Practice, 19, 301-317.

[10] Xie, Y., Zou, T.T. and Jiang, F.X. (2020) Progress of Rehabilitation Nursing in Patients with Constipation after Spinal Cord Injury. Today Nurse, 27, 17-19.

[11] Eberhardt, S., Heinemann, A., Kulp, W., et al. (2004) Health Technology Assessment Report: HTA-Bericht: Dekubitusprophylaxe und -Therapie. DIMDI (Eds.). http://portal.dimdi.de/de/hta/hta_berichte/hta128_bericht_de.pdf

[12] Li, W. (2002) New Progress in Caring Pressure Sores. Journal of Nurses Training, 17, 20-21.

[13] Zhang, Z.G., Cai, X.L., Ma, F.L., et al. (2020) Nursing Care of 37 Cases of Patients with COVID-19 from Different Cultural Backgrounds Imported from Abroad in Lanzhou City. Chinese Journal of Nursing, 55, 837-840. 